\title{
Resetting the Biological Clock: Mediation of Nocturnal CREB Phosphorylation via Light, Glutamate, and Nitric Oxide
}

\author{
Jian M. Ding, ${ }^{1,3}$ Lia E. Faiman, ${ }^{1}$ William J. Hurst, ${ }^{1}$ Liana R. Kuriashkina, ${ }^{2}$ and Martha U. Gillette ${ }^{1,2,3}$ \\ ${ }^{1}$ Department of Cell and Structural Biology, ${ }^{2}$ Molecular and Integrative Physiology, and ${ }^{3}$ The Neuroscience Program, \\ University of Illinois, Urbana, Illinois 61801
}

Synchronization between the environmental lighting cycle and the biological clock in the suprachiasmatic nucleus (SCN) is correlated with phosphorylation of the $\mathrm{Ca}^{2+} / \mathrm{cAMP}$ response element binding protein (CREB) at the transcriptional activating site $\mathrm{Ser}^{133}$. Mechanisms mediating the formation of phosphoCREB (P-CREB) and their relation to clock resetting are unknown. To address these issues, we probed the signaling pathway between light and $\mathrm{P}-\mathrm{CREB}$. Nocturnal light rapidly and transiently induced P-CREB-like immunoreactivity (P-CREB-lir) in the rat SCN. Glutamate (Glu) or nitric oxide (NO) donor administration in vitro also induced $\mathrm{P}$-CREB-lir in SCN neurons only during subjective night. Clock-controlled sensitivity to phase resetting by light, Glu, and NO is similarly restricted to subjective night. The effects of NMDA and nitric oxide synthase (NOS) antagonists on Glu-mediated induction of P-CREB-lir paralleled their inhibition of phase shifting. Significantly, among neurons in which P-CREB-lir was induced by light were NADPH-diaphorase-positive neurons of the SCN's retinorecipient area. Glu treatment increased the intensity of a $43 \mathrm{kDa}$ band recognized by anti-P-CREB antibodies in subjective night but not day, whereas anti- $\alpha$ CREB-lir of this band remained constant between night and day. Inhibition of NOS during Glu stimulation diminished the anti-P-CREB-lir of this $43 \mathrm{kDa}$ band. Together, these data couple nocturnal light, Glu, NMDA receptor activation and NO signaling to CREB phosphorylation in the transduction of brief environmental light stimulation of the retina into molecular changes in the SCN resulting in phase resetting of the biological clock.

Key words: suprachiasmatic nucleus; circadian rhythm; glutamate; NMDA; nitric oxide; CREB phosphorylation; immunocytochemistry; Western blot; nuclear protein extraction; diaphorase; APV; L-NAME; D-NAME; SNAP
The daily rhythms of life are generated by a biological clock, which in mammals resides within the suprachiasmatic nucleus (SCN) of the hypothalamus (Meijer and Rietveld, 1989; Morin, 1994). Although the time-generating mechanism is endogenous to the SCN, the dominant regulator of circadian clock phasing is environmental light. Photic signals are communicated via the retinohypothalamic tract, a direct neural projection from the retina to the SCN (Moore and Lenn, 1972; Johnson et al., 1988). The clock restricts its own sensitivity to stimulation (Gillette et al., 1995) so that only nocturnal light adjusts its timing, causing phase delays in early night and phase advances in late night (DeCoursey, 1960; Daan and Pittendrigh, 1976; Takahashi and Zatz, 1982; Summers et al., 1984). This ensures that daily behavioral rhythms are synchronized appropriately to phases of the environmental cycle of darkness and light. We sought to discriminate the multiple events linking sensory stimulation with SCN changes that orchestrate these behavioral modulations.

\footnotetext{
Received July 7, 1996; revised Oct. 25, 1996; accepted Oct. 29, 1996.

This study was supported by Public Health Service Grants NS22155 and NS33240 from the National Institute of Neurological Diseases and Stroke. We are indebted to D. Ginty, J. Kornhauser, and M. Greenberg for technical advice and their generous gifts of antibodies and CREBtide used in this study; S. Rogers and B. Carragher of the Optical Visualization Facility of the Beckman Institute for Advanced Science, UIUC; W. Greenough for advice on quantitation of immunocytochemistry; and M. Churchill, D. Clayton, and A. Nardulli for the technical advice and critical reading of an early version of this manuscript.

Correspondence should be addressed to Dr. Martha U. Gillette, Department of Cell and Structural Biology, B107 Chemistry and Life Science Laboratory, 601 South Goodwin Avenue, University of Illinois, Urbana, IL 61801.

Copyright (C) 1997 Society for Neuroscience $0270-6474 / 97 / 170667-09 \$ 05.00 / 0$
}

Important features of the photic phase shifting process are (1) the brevity of the light pulse that can permanently reset the near $24 \mathrm{hr}$ cycle of circadian clocks (DeCoursey, 1960; Daan and Pittendrigh, 1976; Takahashi and Zatz, 1982) and (2) alterations of transcriptional elements (Ginty et al., 1993). Within 2 min, a phase-resetting light stimulus induces transcriptional changes in a state variable, the frequency gene, of the Neurospora clock (Crosthwaite et al., 1995). In the nervous system, too, long-lasting changes induced by a brief stimulus often involve the alteration of gene expression (Goelet et al., 1986; Montarolo et al., 1986; Morgan and Curran, 1989; Sheng and Greenberg, 1990; Alberini et al., 1994). Induction of immediate-early genes, especially members of the fos and jun families, occurs in the SCN within $1 \mathrm{hr}$ of a photic stimulus that induces phase shifts of circadian rhythms (Rea, 1989; Rusak et al., 1990; Kornhauser et al., 1992; Takeuchi et al., 1993). Neurotransmission is coupled to gene induction in neurons via signaling cascades that activate DNA-binding proteins through transient phosphorylation of transcriptional activating amino acid residues. Brief exposure of hamsters to light at night induces phosphorylation of such a protein, cAMP response element binding protein (CREB), at its transactivation site; Ser ${ }^{133}$-phosphorylated CREB (P-CREB) appears in the SCN within $5 \mathrm{~min}$ on light exposure (Ginty et al., 1993). This duration of light induces robust phase shifts of the circadian rhythm of locomotor activity in the days after stimulation. Thus, P-CREB is the earliest sign in the SCN of transcriptional activation by photic stimulation that leads to adjustments in 24 hr timing. 
Although the sequence of events by which light signals P-CREB formation is unknown, essential components of the pathway mediating light-stimulated phase resetting have been identified. Light induces clock resetting through an excitatory signal transduction pathway mediated by glutamate (Glu), NMDA receptor activation, stimulation of nitric oxide synthase (NOS), and intercellular movement of nitric oxide (NO) (Ding et al., 1994b; Shibata et al., 1994; Shirakawa and Moore, 1994; Watanabe et al., 1994). In cultured hippocampal neurons and PC-12 cells, Glu activation of NMDA receptors with subsequent $\mathrm{Ca}^{2+}$ influx rapidly induces phosphorylation of CREB (Bading et al., 1993; Gallin and Greenberg, 1995; Ghosh and Greenberg, 1995). Because light triggers P-CREB in the SCN and the Glu/NO pathway mediates light-induced phase shifts, we examined the hypothesis that Glu and NO are components of the signal transduction cascade that activates CREB in the circadian clock.

To selectively probe elements regulating CREB phosphorylation, we compared the response of the SCN in vivo to light with that in vitro to specific reagents affecting Glu and NO pathways. We used the rat $\mathrm{SCN}$ in a hypothalamic brain slice, a preparation in which the circadian clock persists for $3 \mathrm{~d}$ (Gillette, 1991). The mean firing frequency of the population of SCN neurons undergoes a $24 \mathrm{hr}$ oscillation in vitro (Green and Gillette, 1982) that matches the pattern of SCN neuronal activity in vivo (Inouye and Kawamura, 1979, 1982). Likewise, the SCN clock in vitro continues to regulate its own sensitivity to stimuli that can adjust its timing over the circadian cycle (Ding et al., 1994b; Gillette et al., 1995; Gillette, 1996).

\section{MATERIALS AND METHODS}

Brain slice preparation and electrophysiological recordings of the SCN circadian neuronal activity. The detailed description of this method has been reported previously (Gillette, 1991; Ding et al., 1994b). Briefly, a $500-\mu \mathrm{m}$-thick coronal hypothalamic brain slice containing the paired SCN was prepared at least $2 \mathrm{hr}$ before the onset of the dark phase from 7- to 10-week-old, inbred Long-Evans rats housed in a 12:12 hr light/dark cycle. The brain slices survived for up to $3 \mathrm{~d}$ in vitro with continuous perifusion of Earle's Essential Balanced Salt Solution (EBSS, Life Technologies, Gaithersburg, MD), supplemented with $24.6 \mathrm{~mm}$ glucose, 26.2 $\mathrm{mm}$ sodium bicarbonate, and $5 \mathrm{mg} / \mathrm{l}$ of gentamicin, and saturated with $95 \% \mathrm{O}_{2} / 5 \% \mathrm{CO}_{2}$ at $37^{\circ} \mathrm{C}, \mathrm{pH}$ 7.4. The single-unit activity of the SCN neurons was recorded extracellularly with a glass microelectrode, and running means were calculated to determine the time-of-peak activity. The unperturbed sinusoidal pattern of neuronal activity is predictably high in the day and low during the night, peaking at mid-day at approximately circadian time 7 (CT 7) (Prosser and Gillette, 1989). The onset of the light phase of the entraining light/dark cycle of the brain slice donor was designated as CT 0 . Thus, the time-of-peak of the neuronal firing rate can be used as a reliable assessment of the phase of the circadian rhythm (Gillette et al., 1995). For treatment of the brain slice, the perifusion pump was stopped, and a $0.2 \mu \mathrm{l}$ microdrop of a test substance dissolved in EBSS was applied bilaterally to the SCN for $10 \mathrm{~min}$ before rinsing with EBSS and resuming pumping with normal medium. To evaluate potential blockers of the stimulus, the bathing medium was replaced with antagonists dissolved in EBSS, $\mathrm{pH} 7.4$, for 20 min before the phase-shifting stimulus was applied to the SCN. To determine the phase of the circadian rhythm, the time-of-peak neuronal activity was assessed for the following 1 or $2 \mathrm{~d}$ in vitro.

Immunocytochemistry and histochemistry. We compared the sensitivity of this biological clock to treatments during the subjective day, when the clock is insensitive to light-induced resetting, with its responsiveness during the subjective night. Reagents were applied directly to the SCN in vitro, and their efficacy was assessed with reference to that of light in vivo. The appearance of P-CREB in the tissue was assayed using an antibody (anti-P-CREB) that recognizes the peptide sequence containing phosphorylated $\operatorname{Ser}^{133}$. This sequence is found within the transcriptional activating domain of the CREB family of transcription factors $\alpha \mathrm{CREB}$, CREM, and ATF-1 (Ginty et al., 1993; Ghosh et al., 1994).
To assess Glu-induced P-CREB-like immunoreactivity (P-CREB-lir) in vitro, Glu was applied to the SCN for $10 \mathrm{~min}$, after which the brain slices were placed in $4 \%$ paraformaldehyde for $12-18 \mathrm{hr}$ at $4^{\circ} \mathrm{C}$. The slices were then transferred to a cryoprotectant (15\% sucrose in PBS) for $24 \mathrm{hr}$ before sectioning at $20 \mu \mathrm{m}$ by cryostat at $-15^{\circ} \mathrm{C}$; sections were affixed to gelatin-coated microscope slides and stored at $-15^{\circ} \mathrm{C}$. For light-induced P-CREB-lir, animals were exposed to 150 lux of white light for $10 \mathrm{~min}$ at CT 19. After appropriate durations, animals were deeply anesthetized with sodium pentobarbital $(75 \mathrm{mg} / \mathrm{kg}$, i.p.) and perfused intracardially with $60 \mathrm{ml}$ of chilled PBS followed by $600 \mathrm{ml}$ of chilled $4 \%$ paraformaldehyde. For immunocytochemical detection of P-CREB, the tissue sections were rinsed with PBS and then incubated for $1 \mathrm{hr}$ at room temperature with $0.3 \%$ Triton X-100 and $1 \%$ heat-inactivated goat serum to permeabilize the lipid membrane and block nonspecific binding sites, respectively. The sections were then incubated for $18-24 \mathrm{hr}$ at $4^{\circ} \mathrm{C}$ with affinity-purified polyclonal anti-P-CREB antibody diluted 1:1000 in PBS with $0.3 \%$ Triton X-100 detergent. The avidin-biotin-peroxidase complex (Vector Laboratories, Burlingame, CA) was used with the glucose oxidase reaction as the peroxide generator to form an insoluble brown $\mathrm{DAB}$ product localizing the antigen. The sections were then air-dried before alcohol dehydration and xylene defatting, and coverslips were applied with Permount (Ding et al., 1994a).

Because neuronal NOS also mediates the neuronal NADPHdiaphorase histochemical reaction (Hope et al., 1991), NADPHdiaphorase staining is widely used to visualize neuronal NOS. Histochemical detection of neuronal NADPH diaphorase was performed according to Vincent and Kimura (1992) with minor modifications. Briefly, the rat brains were fixed and sectioned as above for immunocytochemistry. The tissue sections were first incubated in PBS with $0.3 \%$ Triton X-100 for 30 $\mathrm{min}$ at room temperature and then incubated with $0.5 \mathrm{~mm}$ nitro blue tetrazolium (NBT, Sigma, St. Louis, MO) and $1.0 \mathrm{~mm} \beta$-NADPH (Sigma) in $50 \mathrm{~mm}$ Tris $-\mathrm{HCl}$ buffer, $\mathrm{pH} 7.8$, at $37^{\circ} \mathrm{C}$ for $30-60 \mathrm{~min}$. The tissue sections were transferred to PBS to stop the color reaction. Histochemical reactions in the absence of NADPH or NBT, respectively, were used as controls.

Because P-CREB is a nuclear protein and NOS/diaphorase is a cytoplasmic enzyme, it is possible to discriminate the two proteins in the same neurons to evaluate their colocalization. For double-label immunocytochemistry and histochemistry, the animals were exposed to a light pulse for $10 \mathrm{~min}$ at CT 19. The animals were perfused, and the brains were removed and sectioned as described above. The tissue sections were first incubated with the primary and secondary antibodies recognizing P-CREB to generate a brown DAB-peroxidase staining in the nucleus. After PBS washes, the sections were then incubated with NADPH and NBT. A diaphorase histochemical reaction will generate blue NBT staining in the cytoplasm. The sections were then dehydrated and mounted as described above.

Quantitative analysis of P-CREB-lir. Because the tissue fixation and immunocytochemical reaction may vary from time to time, all experimental and control slices were processed simultaneously with the same batch of reagents. Cell counting was performed manually to include all visible P-CREB-lir-positive cells within one focal plane of each $50 \mu \mathrm{m}$ section of the entire SCN. For the supraoptic nucleus (SON), only the sections that were on the same level of the nucleus circularis were counted. For computerized imaging analysis of P-CREB-lir, brain sections were placed under a Zeiss microscope, and the images were captured by a CCD video camera (NEC). The digitalized image was displayed on a Macintosh computer equipped with National Institutes of Health Image software. By moving a cursor masking the SCN, the image intensity in nonresponsive brain regions was determined. A frequency histogram of the image intensity was used to select a threshold cutoff for signal from background. By calculating the histogram for image intensity above the threshold, the average intensity of the signal and the number of pixels exceeding the threshold were determined (data not shown). Statistical analyses were performed using the software SAS.

Nuclear protein extraction and Western blot. Brain slices were maintained in vitro for $>5 \mathrm{hr}$ until the appropriate CT, when they were quick-frozen on a glass slide cooled on dry ice. A stainless steel needle (400 $\mu \mathrm{m}$ inner diameter) was used to punch both SCN from each $500-\mu \mathrm{m}$-thick brain slice. For each experimental condition, the SCN from five animals were collected $\left(\sim 50 \mu \mathrm{g}\right.$ protein) and stored at $-80^{\circ} \mathrm{C}$ until use. Samples enriched for nuclear protein were prepared according to Dash et al. (1995). All steps were carried out at $4^{\circ} \mathrm{C}$ with the following reagents present in all solutions: $1 \mathrm{~mm}$ EGTA, 5 mM EDTA, $2 \mathrm{~mm} \mathrm{NaF}$, $1 \mathrm{~mm}$ sodium orthovanadate, $10 \mathrm{~mm}$ glycerol phosphate, $200 \mu \mathrm{M}$ sodium 


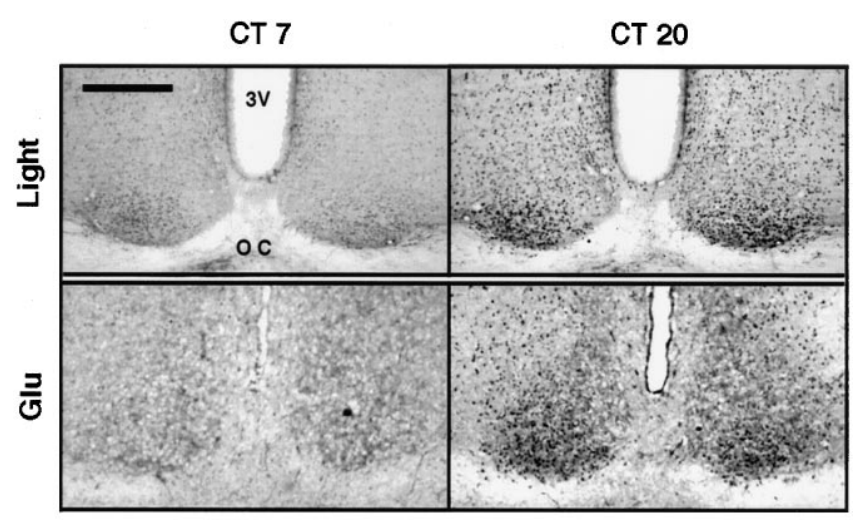

Figure 1. Sensitivity of SCN to CREB phosphorylation by light or Glu is restricted to the night. Robust nuclear staining of P-CREB-lir was induced in the SCN under free-running conditions after stimulation with light in vivo (150 lux, $10 \mathrm{~min})$ or Glu in vitro $(10 \mathrm{~mm}$ in $0.2 \mu \mathrm{l}$ drop, $10 \mathrm{~min})$ during subjective night (CT 19-20). In contrast, only a weak basal level of P-CREB-lir was detected at CT 7 after the same light or Glu treatment. When the anti-P-CREB antibody was preabsorbed with equal amount of phospho-CREBtide, no positive immunoreactivity was detected. Scale bar, $200 \mu \mathrm{m}$.

pyrophosphate, $5 \mu \mathrm{M}$ microcystin, $0.5 \mu \mathrm{g} / \mathrm{ml}$ leupeptin, $0.7 \mu \mathrm{g} / \mathrm{ml}$ pepstatin, $1.0 \mu \mathrm{g} / \mathrm{ml}$ aprotinin, $40 \mu \mathrm{g} / \mathrm{ml}$ bestatin, and $1 \mathrm{~mm}$ PMSF. The SCN tissue was washed with sucrose buffer $(0.25 \mathrm{M}$ sucrose, $15 \mathrm{mM}$ HEPES, 60 $\mathrm{mm} \mathrm{KCl}, 10 \mathrm{~mm} \mathrm{NaCl}, \mathrm{pH} 7.2$ ), and centrifuged at $2000 \times \mathrm{g}$ for $10 \mathrm{~min}$. Pellets were resuspended in cell lysis buffer $\left(1.5 \mathrm{~mm} \mathrm{MgCl}_{2}, 10 \mathrm{~mm} \mathrm{KCl}\right.$, $15 \mathrm{~mm}$ HEPES, $\mathrm{pH}$ 7.2), agitated gently, and centrifuged at $4000 \times g$ for $10 \mathrm{~min}$. Pellets were resuspended in $1 \mu \mathrm{l} / \mathrm{SCN}$ of nuclear lysis buffer $(1.5$

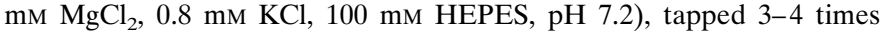
during a $30 \mathrm{~min}$ agitation, and centrifuged at $14,000 \times \mathrm{g}$ for $30 \mathrm{~min}$. Supernatants containing the enriched nuclear extract were then stored at $-80^{\circ} \mathrm{C}$.

For Western blots, denaturing buffer was added to a $2 \mu \mathrm{l}$ aliquot of SCN nuclear extract from each treatment. Samples were subjected to SDS-PAGE on a $12 \%$ gel, transferred to nitrocellulose, and probed as in Ginty et al. (1993), with the exception that a horseradish peroxidase linked to goat anti-rabbit secondary (1:1000) and an ECL fluorescence system (Amersham, Arlington Heights, IL) were used for detection. To compare intersample protein loading, gels were silver-stained (Morrissey, 1981), and each lane was scanned densitometrically.

\section{RESULTS}

\section{Circadian sensitivities of the SCN to Glu- and light-induced CREB phosphorylation are coincident}

To evaluate the temporal sensitivities of the SCN to Glu- and light-induced P-CREB formation, we compared the effects of these stimuli in subjective day and night. Induction of P-CREB-lir in the SCN was dependent on the CT of treatment. When Glu (0.2 $\mu l$ drop at $10 \mathrm{~mm}$ ) was applied to the surface of the SCN for 10 min during the subjective day (CT 6-7), no increase in P-CREBlir above basal level was observed. In contrast, during the subjective night (CT 19-20), this treatment induced robust nuclear P-CREB-lir within the SCN $(n=8)$ (Fig. 1). Application of a microdrop of the medium only at CT 20 for $10 \mathrm{~min}$ did not change P-CREB-lir from the basal level $(n=6)$. Staining was fully blocked by preincubating the antibody with P-CREBtide.

When the efficacy of Glu applied in vitro was assessed with reference to that of light in vivo, we observed that the patterns of P-CREB-lir were similar (Fig. 1), despite differences in the spatial character of stimulation. After a 10 min exposure of rats

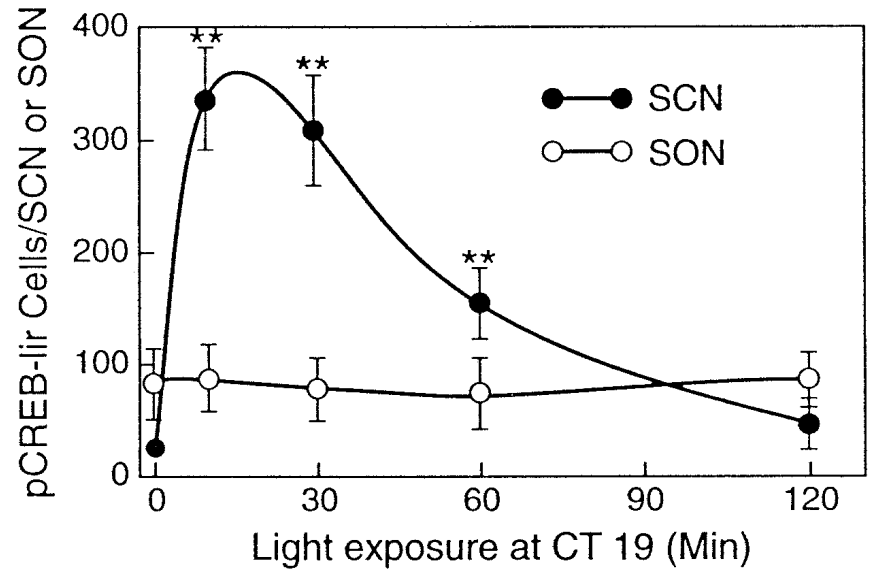

Figure 2. Time course of P-CREB-lir in the SCN after light exposure. After a light pulse at CT 19, P-CREB-lir in the SCN reached maximum level within $10 \mathrm{~min}$ and remained at this level at $30 \mathrm{~min}$. It declined to approximately half-maximum by $1 \mathrm{hr}$ and returned to nearly basal level in $\sim 2 \mathrm{hr}$. In contrast, the P-CREB-lir in the SON of the same animals remained unchanged throughout the entire duration after the light exposure. General linear regression (GLM) for unbalanced ANOVA and post hoc test (Duncan) revealed that the levels of P-CREB-lir in the SCN at 10, 30 , and $60 \mathrm{~min}$ groups are significantly different from basal level and from each other except for the values at 10 and $30 \mathrm{~min}$. Each data point represents at least three animals; ${ }^{*} p<0.01$.

to light, distinctive nuclear staining of neurons was localized primarily in the ventrolateral region of the $\mathrm{SCN}$, where projections from the retina terminate. However, P-CREB-lir induced by Glu in the brain slice was not always restricted to the ventral SCN, possibly because the microdrop can exceed the retinorecipient region. Furthermore, despite the less localized P-CREB staining, the phase shifts induced by Glu microdrops are similar in timing, direction, and amplitude to those induced by light.

\section{Induction of P-CREB-lir in the SCN after light exposure is rapid and transient}

To establish a time course for the level of light-induced P-CREBlir in the SCN, animals were killed at 10, 30, 60, and 120 min after the 10 min light exposure at CT 19. Quantitative analysis of P-CREB-lir was performed by cell counting as well as by computerized imaging analysis. Although immunocytochemical methods cannot determine the exact amount of antigen, they do permit the measurement of relative changes between control and treated groups, while preserving neuroanatomical localization (Ding et al., 1994a; Mize, 1994). Basal P-CREB-lir was low in the rat SCN, similar to basal levels in the hamster (Ginty et al., 1993). However, a high level of P-CREB-lir appeared rapidly in response to a light stimulus at night (Fig. 2). Quantitative analysis of P-CREB-lir in the SCN showed that after a light pulse at CT 19, P-CREB-lir reached maximum level within $10 \mathrm{~min}$ and remained at this level at $30 \mathrm{~min}$. It declined to approximately half-maximum by $1 \mathrm{hr}$, and returned to nearly basal level in $\sim 2 \mathrm{hr}(n=18)$ (Fig. 2). In contrast to the SCN, the level of P-CREB-lir in the supraoptic nucleus SON of the same animals was unaffected by light exposure. 




Figure 3. Blockade of Glu-induced P-CREB-lir in the SCN brain slices by inhibitors of the NMDA receptor and NOS. A 20 min preincubation in the NMDA receptor blocker APV (0.1 mM) or the NOS inhibitor, L-NAME $(0.1 \mathrm{~mm})$ diminished Glu-induced P-CREB-lir in the SCN at CT 20, whereas D-NAME $(0.1 \mathrm{mM})$, the inactive stereoisomer of L-NAME, failed to block Glu-induced P-CREB-lir. These levels of staining are representative of four to six replications of each condition. Scale bar, $200 \mu \mathrm{m}$.

\section{Glu-induced CREB phosphorylation in the SCN is channeled via the NMDA receptor and NO signaling pathway}

To determine whether Glu effects on P-CREB were mediated via an NMDA receptor, SCN slices were bathed in a specific NMDA receptor antagonist, 2-amino-5-phosphonovaleric acid (APV, 0.1 $\mathrm{mm}$ ), for 20 min before Glu treatment. Although APV alone had no effect on the basal level of P-CREB-lir $(n=6)$ (Fig. 3), it prevented Glu-induced P-CREB-lir at CT $20(n=6)$. Thus, as in Glu-stimulated phase resetting (Ding et al., 1994b), P-CREB formation requires NMDA receptor activation.

To test whether NOS activation is in the pathway leading to P-CREB induction, a competitive NOS inhibitor, $\mathrm{L}-N^{\mathrm{G}}$-nitro-Argmethyl ester (L-NAME) was used. L-NAME has been shown to block both GLU-induced phase shifts of SCN neuronal circadian rhythms in vitro and light-induced phase changes of locomotory rhythms in vivo (Ding et al., 1994b; Weber et al., 1995a). Preincubation in L-NAME (0.1 $\mathrm{mM})$ for 20 min significantly diminished the P-CREB-lir normally induced by Glu at CT $20(n=8)$, whereas the inactive stereoisomer $\mathrm{D}-N^{\mathrm{G}}$-nitro-Arg-methyl ester (D-NAME, $0.1 \mathrm{~mm}$ ) failed to prevent the effect of Glu on P-CREB-lir $(n=6)$ (Fig. 3). Neither L-NAME nor D-NAME, when applied alone, had apparent effects $(n=6$ each).

Microdrops of an exogenous NO generator, $S$-nitroso- $N$-acetylpenicillamine (SNAP; $0.01 \mathrm{mM}$ ), induced P-CREB-lir in the SCN during the subjective night but not the subjective day $(n=4)$ (Fig. 4). This coincides with the circadian sensitivity of the SCN to NO-induced phase resetting. The pattern of P-CREB-lir induced



Figure 4. CT-dependent P-CREB induction by exogenous NO donor in the SCN. A microdrop $(0.2 \mu \mathrm{l})$ of NO generator SNAP $(0.01 \mathrm{mM})$ applied directly to the SCN induced P-CREB-lir at CT $19(A)$, but not at CT $7(B)$. Nissl stain demonstrates the histological boundary of the rat SCN $(C)$. Scale bar, $200 \mu \mathrm{m}$.

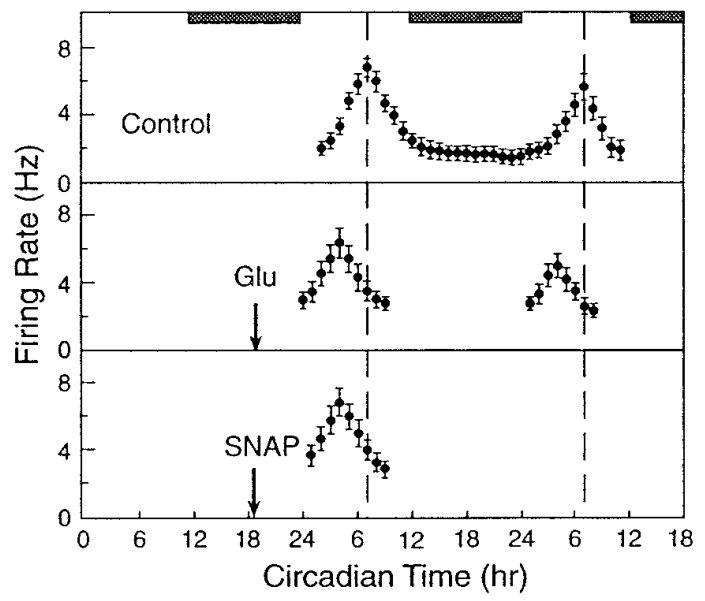

Figure 5. Circadian sensitivity of SCN to phase resetting. Phase resetting was assessed by measuring the time-of-peak of the endogenous circadian rhythms of the neuronal activity of the SCN in brain slice. Top panel, Continuous single-unit extracellular recording of the unperturbed SCN neuronal activity from 112 units over $38 \mathrm{hr}$. The firing rate of this circadian rhythm peaked in midsubjective day at CT 7, on both days 2 and 3 in vitro. Middle panel, At CT 19, Glu advanced the peak of the SCN activity rhythm by $3 \mathrm{hr}$. A $0.2 \mu \mathrm{l}$ droplet of $10 \mathrm{~mm}$ Glu was directly applied to the SCN for 10 min (arrow), followed by EBSS wash. Neuronal activity was recorded over $10-12 \mathrm{hr}$ on each of the next two cycles to define the time-of-peak activity. Bottom panel, At CT 19, a $0.2 \mu \mathrm{l}$ droplet of $0.01 \mathrm{~mm}$ SNAP advanced the peak of the SCN firing rate by $3.5 \mathrm{hr}$. The horizontal bars indicate the subjective night of the circadian cycle. The dashed vertical lines mark the time (CT 7) of the normal peak of the circadian rhythm of the neuronal activity in unperturbed and EBSS-treated controls. 

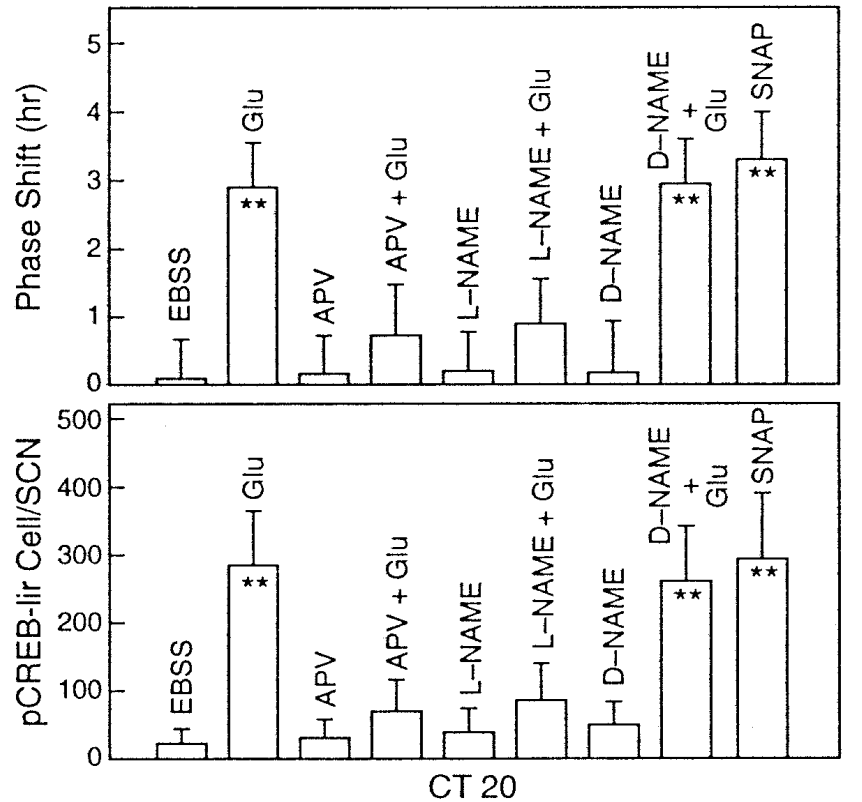

Figure 6. Quantitative comparison of phase shifts and P-CREB-lir induced by various reagents affecting the Glu/NMDA receptor/NOS/NO pathway at CT 19-20. All measurements were made on SCN studied in vitro. Phase shifting data are replotted from Ding et al. (1994b) to facilitate direct comparison. P-CREB-lir was determined after treatment, fixation, and sectioning so that $n=$ each SCN brain slice per treatment (1 SCN slice was obtained from each animal). GLM for unbalanced ANOVA and post hoc test (Duncan) revealed that only Glu, SNAP, and Glu + D-NAME significantly induced phase shifts as well as increased P-CREB-lir from basal levels in the SCN in vitro. Each data point represents the mean \pm SD of four to eight animals; ${ }^{*} p<0.01$.

by SNAP was similar to that induced by Glu, except that the P-CREB-like nuclear staining was sometimes also found outside the $\mathrm{SCN}$, possibly because NO can diffuse to a greater radius than the microdrops. However, the P-CREB-lir inside the SCN was always stronger than the surrounding hypothalamus (Fig. 4).

To compare the effect of Glu and SNAP on phase resetting, single-unit recording was performed to determine the time-ofpeak of the SCN neuronal activity. At CT 19, the phase advance $(3.5 \pm 0.79 \mathrm{hr}, n=4)$ induced by a microdrop of $\operatorname{SNAP}(0.01 \mathrm{~mm})$ applied for $10 \mathrm{~min}$ was overlapping with the response to Glu (3.3 $\pm 0.6 \mathrm{hr}, n=6)$ (Fig. 5).

To evaluate the effects of stimulating or inhibiting elements of the Glu, NMDA, and NO signaling pathway on both production of P-CREB-lir and phase shifts, the results of each treatment were compared quantitatively (Fig. 6). Only in SCN exposed to Glu, SNAP, and Glu + D-NAME were there significant phase shifts and P-CREB-induction $(p<0.01)$ (Fig. 6). This demonstrates that both the stimulatory effects of Glu, NMDA, and NO donors and the inhibitory effects of NMDA and NOS antagonists on Glu induction of P-CREB-lir matched their effects on SCN clock phasing ( $n=4-6$ per condition) (Fig. 6). Together, these data reveal a quantitative correlation between phase shifts of the circadian rhythm and putative P-CREB formation in the SCN during the nocturnal period of sensitivity of the clock to light-, Glu-, and NO- stimulated resetting.



Figure 7. Colocalization of NADPH diaphorase and light-induced P-CREB-lir in the SCN. NADPH-diaphorase-positive neurons of varying size, arborization, and intensity are localized in the ventrolateral region of the SCN $(a)$. Some diaphorase-containing neurons in the ventrolateral SCN have long processes projecting to the core of the SCN $(b)$. The double-label immunocytochemistry and histochemistry revealed that the brown nuclear staining of P-CERB-lir was colocalized with the blue cytoplasmic staining of neuronal diaphorase within $\mathrm{SCN}$ neurons $(c)$. Scale bars: $a, 50 \mu \mathrm{m} ; b, 40 \mu \mathrm{m} ; c, 20 \mu \mathrm{m}$.

\section{Colocalization of NADPH diaphorase and light-induced P-CREB-lir in the SCN}

To study the spatial relationship between P-CREB- and NOScontaining neurons, a double-label immunocytochemistry and histochemistry procedure was used to evaluate potential colocalization of light-induced P-CREB-lir and NADPH-diaphorase staining in the SCN $(n=12)$. The neuronal NADPH-diaphorase histochemical reaction has been shown to visualize neuronal NOS (Hope et al., 1991). Consistent with previous reports (Decker and Reuss, 1994; Amir et al., 1995; Reuss et al., 1995; Chen et al., 1997), NADPH-diaphorase- and neuronal NOS-containing neurons are sparse in the SCN. They are concentrated in the ventrolateral region (Fig. 7A). SCN neurons of varying size, arborization, and intensity were observed with diaphorase staining (Fig. $7 A, B)$. The double-label histochemistry and immunocytochemistry procedure revealed that the blue cytoplasmic staining of neuronal diaphorase was colocalized in a subset of neurons with the brown nuclear staining of P-CERB-lir after a 10 min light pulse (Fig. $7 C$ ). However, not every diaphorase-containing neuron in 


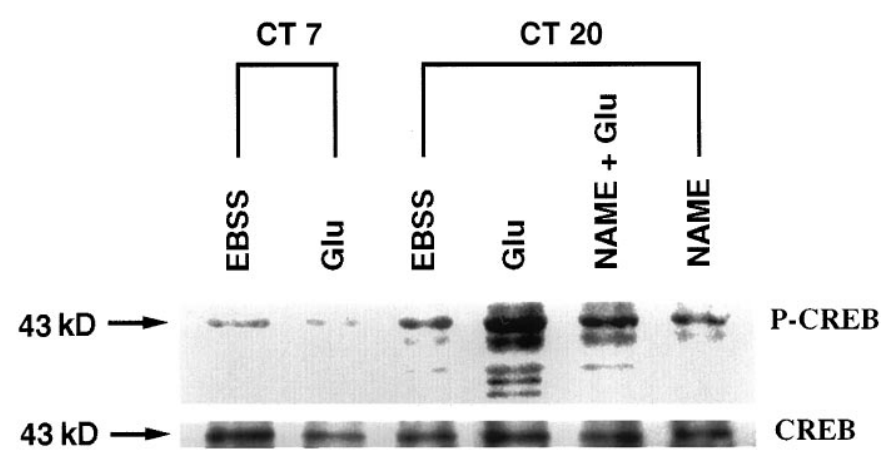

Figure 8. Glu induces a $43 \mathrm{kDa}$ phosphoprotein in the SCN at night. Top panel, Affinity-purified anti-P-CREB antibody was used in Western blot analysis of the SCN nuclear extracts. A robust increase above basal level of a $43 \mathrm{kDa}$ band was detected after Glu treatment at CT 20, but not at CT 7. A less intense reaction was also seen in bands of 33-36 kDa. The NOS inhibitor L-NAME diminished the amount of the $43 \mathrm{kDa}$ band recognized by the anti-P-CREB antibody. Bottom panel, When the same gel was reprobed with the anti- $\alpha$ CREB antibody, equivalent amounts of the 43 $\mathrm{kDa}$ protein were present under all conditions. All experiments were repeated at least three times.

the SCN was colocalized with P-CREB-immunoreactive neuron and visa versa. Both P-CREB-lir neurons and diaphorase-positive neurons were localized in the ventrolateral region of the SCN. However, there were more P-CREB immunoreactive neurons than diaphorase-containing neurons in the SCN. Cell counts of both P-CREB-lir and diaphorase-positive neurons revealed that the ratio between them was $\sim 50: 1$.

\section{Glu induces the appearance of a $43 \mathrm{kDa}$ CREB-positive phosphoprotein in SCN nuclear extracts at night}

To further characterize the protein species representative of P-CREB-lir induced by Glu, SCN nuclear extracts (Dash et al., 1995) were denatured (Laemmli, 1970) and subjected to Western blot analysis with the anti-P-CREB antibody. An increase above basal P-CREB-lir was observed in a $43 \mathrm{kDa}$ nuclear protein after Glu treatment in the subjective night, but not subjective day $(n=6)$ (Fig. 8$)$. In addition to this major band, increased immunoreactivity in minor bands of $33-36 \mathrm{kDa}$ was also observed after night-time treatment. Consistent with the results of quantitative immunocytochemistry, the NOS inhibitor L-NAME greatly diminished the amount of the $43 \mathrm{kDa}$ band recognized by the anti-P-CREB antibody. When the same gel was reprobed with the anti- $\alpha \mathrm{CREB}$ antibody, which recognizes $\alpha \mathrm{CREB}$ regardless of the phosphorylation state, the quantity of this $43 \mathrm{kDa}$ protein was constant across samples and between day and night.

\section{DISCUSSION}

Phosphorylation of the transcription factor CREB has been identified as a key step coupling extracellular stimuli to long-lasting intracellular responses (Sheng and Greenberg, 1990; Brindle and Montminy, 1992). When CREB is phosphorylated on $\mathrm{Ser}^{133}$, by cAMP or $\mathrm{Ca}^{2+} /$ calmodulin-dependent protein kinases (Gonzalez and Montminy, 1989; Dash et al., 1991; Sheng et al., 1991), it becomes active in promoting transcription at the cAMP-response element (CRE) (Hunter and Karin, 1992; Chrivia et al., 1993).
P-CREB can activate the CREs of a number of genes, including the immediate-early gene c-fos (Sassone-Corsi et al., 1988; Sheng et al., 1990) and zif/268 (Sakamoto et al., 1991), and genes encoding synaptic vesicular protein synapsin I (Sauerwald et al., 1990). CREB phosphorylation has been implicated in the learning and memory processes in a wide range of organisms, including Aplysia (Dash et al., 1990; Alberini et al., 1994), Drosophila (Tully et al., 1994; Yin et al., 1994, 1995), and mammals. CREB-deficient mutant mice exhibit impaired long-term memory, whereas shortterm memory remains intact (Bourtchuladze et al., 1994). Phase shifting of the circadian clock is a neuronal phenomenon consistent with this coupling pattern between short-term extracellular stimuli, P-CREB induction, and long-term response with behavioral consequences; a brief light pulse to free-running animals during their subjective night can rapidly induce P-CREB in the $\mathrm{SCN}$ and reset the phase of circadian behavioral cycles (Ginty et al., 1993).

The present study demonstrates that direct application of Glu or an NO-releasing agent to the SCN, treatments that induce phase resetting, can induce phosphorylation of the transcription factor $\mathrm{CREB}$ at the transcriptional regulatory site $\operatorname{Ser}^{133}$. P-CREB was induced only at CTs when the clock is sensitive to phase resetting by light, Glu, and NO. Further, we demonstrate that production of the gaseous neurotransmitter NO, a critical element in light- and Glu-induced phase shifting of circadian rhythms (Ding et al., 1994b; Weber et al., 1995a), is required for the phosphorylation of CREB through the Glu pathway. The $\mathrm{P}$-CREB-lir induced in vitro by Glu possesses the same molecular weight as P-CREB-lir induced by light in vivo.

The time course of P-CREB induction by light in the SCN is rapid and transient; peak P-CREB-lir appears 10-30 min after the onset of the light pulse. It remains significantly elevated at $60 \mathrm{~min}$ but returns to basal level by $120 \mathrm{~min}$. The timing of this response is consistent with findings of P-CREB induction in the magnocellular hypothalamic SON by salt loading (Shiromani et al., 1995) and the parvocellular neurons in the PVN by stress (Kovacs and Sawchenko, 1996), respectively. In addition, a quantitative correlation exists both between phase shifts of circadian rhythms and the level of P-CREB-lir stimulated by light and Glu at CT 20. Because NO is a freely diffusible intercellular messenger and intercellular diffusion is required for the alteration in phase (Ding et al., 1994b), it is likely that a relatively smaller population of NOS-containing neurons may be able to activate a larger population of target cells. We found that Glu-induced P-CREB-lir in $\sim 50$ times more neurons than the number of diaphorasecontaining neurons in the SCN. Furthermore, the extensive spatial overlap and colocalization between light-induced P-CREB-lir and NADPH diaphorase in the ventrolateral retinorecipient region of the SCN support the idea that NOS-containing neurons may be targets of photic entrainment signals from the retina (Decker and Reuss, 1994; Ding et al., 1994b).

Although it remains to be proven whether P-CREB formation is essential for phase shifting of circadian rhythms, it is clear that $\mathrm{P}$-CREB is useful as a marker to study the signal transduction elements linking nocturnal light and Glu/NO elevation to $\mathrm{P}-\mathrm{CREB}$ formation and its molecular consequences. The signaling steps between Glu/NO and P-CREB are presently unknown. However, $\mathrm{Ca}^{2+}$ and/or cGMP are likely to play important roles. NO can potentiate $\mathrm{Ca}^{2+}$-induced gene expression involving the activation of CREB in neuronal cells (Peunova and Enlkolopov, 1993), and NO can stimulate transcription via a cGMP pathway, including expression of immediate-early genes c-fos and jun $\mathrm{B}$ in 
PC-12 cells (Haby et al., 1994). A study of hippocampal neurons demonstrated a functional correlation between CREB phosphorylation and the induction of both long-term potentiation (LTP) and long-term depression (LTD). P-CREB formation triggered by LTP- or LTD-inducing stimuli required calmodulin and $\mathrm{Ca}^{2+}$ calmodulin-dependent protein kinase (CaM kinase), but not cAMP-dependent kinase (PKA) activities (Deisseroth et al., 1996). Both CaM kinase and cGMP-dependent protein kinase (PKG) have been implicated as mediators of light-induced phase shifts in hamster behavioral rhythms (Golombek and Ralph, 1994; Weber et al., 1995b; Mathur et al., 1996), and a CaM kinase inhibitor has recently been reported to attenuate light-induced P-CREB in hamster SCN (Glolombek and Ralph, 1995).

This study has identified the initial signal transduction elements linking photic stimulation to CREB phosphorylation in the SCN. These signaling elements are the same as those that are critical to light-induced phase resetting. The aggregate data support a signaling pathway in which nocturnal light impinging on the retina stimulates glutamate release from terminals of the retinal ganglion cells projecting to the ventrolateral SCN. Necessary elements at SCN neurons include NMDA receptor activation, NOS stimulation, and intercellular movement of NO (Ding et al., 1994). Because both the NMDA and non-NMDA Glu receptors are present in the SCN (Mikkelsen et al., 1993; Gannon and Rea, 1994) and non-NMDA receptor agonists can induce phase shifts (Shibata et al., 1994), non-NMDA glutamatergic neurotransmission may also contribute to stimulating CREB phosphorylation in the SCN. However, it is likely that the influx of $\mathrm{Ca}^{+2}$ attending NMDA receptor activation leads to activation of kinases such as CaM kinase as well as of NOS. The resulting rise in NO, in turn, could activate PKG. The relative contributions of these potential signaling pathways to CREB phosphorylation requires careful evaluation. Nevertheless, the data thus far place P-CREB at the earliest point of the critical transactivational sequence that intersects with the clock mechanism.

Although the target gene(s) of P-CREB in the SCN have yet to be identified, c-fos has been suggested as a candidate (Ginty et al., 1993). Induction of c-fos is regulated by P-CREB in response to $\mathrm{Ca}^{2+}$, cAMP, and growth factors in hippocampal and PC-12 cells (Sheng et al., 1988; Berkowitz et al., 1989; Sheng et al., 1990). In the SCN, P-CREB formation precedes the appearance of c-fos mRNA in response to light (Kornhauser et al., 1992), and c-fos mRNA is induced in proportion to the intensity of the light stimulus (Kornhauser et al., 1990). In addition, intracerebroventricular injection of antisense oligonucleotides for c-fos and jun B attenuates c-fos transcription and light-induced phase shifts (Wollnik et al., 1995). However, a recent study reported an uncoupling between NO-mediated photic stimulation of hamster behavioral phase shifts and Fos expression in the SCN (Weber et al., 1995a). Furthermore, systemic saline injection and handling can induce behavioral phase shifting and Fos expression (Edelstein and Amir, 1995) but not P-CREB-lir (Sumova et al., 1994) in the SCN. Moreover, it was reported recently that light-induced Fos expression in the rat SCN was codistributed but not colocalized with NADPH diaphorase (Amir et al., 1995). Our present study demonstrates that a number of NADPH-diaphorase-positive neurons do colocalize with the light-induced P-CREB-lir in the rat SCN. Therefore, it is likely that multiple cellular components, signal transduction pathways, and transcriptional activations contribute to the SCN response to light.
Finally, the free-running biological clock shows circadian changes in vivo and in vitro in its response to light, Glu, and NO (Ding et al., 1994b). Therefore, the clock controls its own temporal sensitivity to these stimuli (Gillette, 1996). The consequence is that P-CREB formation and phase resetting can only be stimulated through this signaling pathway in the nocturnal domain of the clock's cycle. Yet, NMDA receptors (Gannon and Rea, 1994), NOS (Chen et al., 1997), and CREB (Ginty et al., 1993) each are present in both night and day. Therefore, among the signaling elements between NO and CREB must lie the clock-controlled molecular gate(s) through which the clock regulates its own temporal sensitivity to these resetting stimuli.

\section{REFERENCES}

Alberini CM, Ghiradi M, Metz R, Kandel ER (1994) C/EBP is an immediate-early gene required for the consolidation of long-term facilitation. Cell 76:1099-1114.

Amir S, Robinson B, Edelstein K (1995) Distribution of NADPHdiaphorase staining and light-induced Fos expression in the rat suprachiasmatic nucleus region supports a role for nitric oxide in the circadian system. Neuroscience 69:545-555.

Bading H, Ginty DD, Greenberg ME (1993) Regulation of gene expression in hippocampal neurons by a distinct calcium signaling pathway. Science 260:181-186.

Berkowitz LA, Riabowol KT, Gilman MZ (1989) Multiple sequence elements of a single functional class are required for cyclic AMP responsiveness of the mouse c-fos promoter. Mol Cell Biol 9:4272-4281.

Bourtchuladze R, Frenguelli B, Blendy J, Cioffi D, Schutz G, Silva AJ (1994) Deficient long-term memory in mice with a targeted mutation of the cAMP-responsive element-binding protein. Cell 79:59-68.

Brindle PK, Montminy MR (1992) The CREB family of transcription activators. Curr Opin Genet Dev 2:199-204.

Chen D, Hurst WJ, Ding JM, Faiman LE, Mayer B, Gillette MU (1997) Localization and characterization of the nitric oxide synthase in the rat suprachiasmatic nucleus: evidence for a prominent nitrergic plexus in the biological clock. J Neurochem, in press.

Chrivia JC, Kwok RPS, Lamb N, Nagiwara M, Montminy MR, Goodman RH (1993) Phosphorylated CREB binds specifically to the nuclear protein CBP. Nature 365:855-859.

Crosthwaite SK, Loros JJ, Dunlap JC (1995) Light-induced resetting of a circadian clock is mediated by a rapid increase in frequency transcript. Cell 81:1003-1012.

Daan S, Pittendrigh CS (1976) A functional analysis of circadian pacemakers in nocturnal rodents. II. The variability of phase response curves. J Comp Physiol 106:223-237.

Dash PK, Hochner B, Kandel ER (1990) Injection of the cAMPresponsive element into the nucleus of Aplysia sensory neurons blocks long-term facilitation. Nature 345:718-721.

Dash PK, Karl KA, Colicos MA, Prywes R, Kandel ER (1991) cAMP response element-binding protein is activated by $\mathrm{Ca}^{2+}$-calmodulin as well as cAMP-dependent protein kinase. Proc Natl Acad Sci USA 88:5061-5065.

Dash PK, Moore AN, Dixon CE (1995) Spatial memory defects, increased phosphorylation of the transcription factor CREB, and induction of the AP-1 complex following experimental brain injury. J Neurosci 15:2030-2039.

Decker K, Reuss S (1994) Nitric oxide-synthesizing neurons in the hamster suprachiasmatic nucleus. A combined NOS- and NADPH-staining and retinohypothalamic tract tracing study. Brain Res 666:284-288.

DeCoursey PJ (1960) Daily light sensitivity rhythm in a rodent. Science 131:33-35.

Deisseroth K, Bito H, Tsien RW (1996) Signaling from synapse to nucleus: postsynaptic CREB phosphorylation during multiple forms of hippocampal synaptic plasticity. Neuron 16:89-101.

Ding JM, Carver WC, Terracio L, Buggy J (1994a) Proto-oncogene c-fos and the regulation of vasopressin gene expression during dehydration. Mol Brain Res 21:247-255.

Ding JM, Chen D, Weber ET, Faiman LE, Rea MA, Gillette MU (1994b) Resetting the biological clock: mediation of nocturnal circadian shifts by glutamate and NO. Science 266:1713-1717. 
Edelstein K, Amir S (1995) Non-photic manipulations induce expression of Fos protein in the suprachiasmatic nucleus and intergeniculate leaflet in the rat. Brain Res 690:254-258.

Gallin WJ, Greenberg ME (1995) Calcium regulation of gene expression in neurons: the mode of entry matters. Curr Opin Neurobiol 5:367-374.

Gannon RL, Rea MA (1994) In situ hybridization of antisense mRNA oligonucleotides for AMPA, NMDA and metabotropic glutamate receptor subtypes in the rat suprachiasmatic nucleus at different phases of the circadian cycle. Mol Brain Res 23:338-344.

Ghosh A, Greenberg ME (1995) Calcium signaling in neurons: molecular mechanisms and cellular consequences. Science 268:239-247.

Ghosh A, Ginty DD, Bading H, Greenberg ME (1994) Calcium regulation of gene expression in neuronal cells. J Neurobiol 25:294-303.

Gillette MU (1991) SCN electrophysiology in vitro: rhythmic activity and endogenous clock properties. In: Suprachiasmatic nucleus: the mind's clock (Klein DC, Moore RY, Reppert SM, eds), pp 125-143. New York: Oxford UP.

Gillette MU (1996) Regulation of entrainment pathways by the suprachiasmatic circadian clock: sensitivities to second messengers. In: Hypothalamic integration of circadian rhythms. Progress in brain research 111 (Buijs R, Romijn H, Pennartz C, Mirmiran M, eds), pp 119-130. Amsterdam: Elsevier.

Gillette MU, Medanic M, McArthur AJ, Liu C, Ding JM, Faiman LE, Weber ET, Tcheng TK, Gallman EA (1995) Intrinsic neuronal rhythms in the suprachiasmatic nuclei and their adjustment. In: Circadian clocks and their adjustment (Ciba Foundation Symposium 183), pp 134-153. Chichester, UK: Wiley.

Ginty DD, Kornhauser JM, Thompson MA, Bading H, Mayo KE, Takahashi JS, Greenberg ME (1993) Regulation of CREB phosphorylation in the suprachiasmatic nucleus by light and a circadian clock. Science 260:238-241.

Goelet P, Castellucci VF, Schacher S, Kandel ER (1986) The long and the short of long-term memory-a molecular framework. Nature 322:419-422.

Golombek DA, Ralph MR (1994) KN-62, an inhibitor of $\mathrm{Ca}^{++} /$calmodulin kinase II, attenuates circadian responses to light. NeuroReport 5:1638-1640.

Golombek DA, Ralph MR (1995) Circadian responses to light: the calmodulin connection. Neurosci Lett 192:101-104.

Gonzalez GA, Montminy MR (1989) Cyclic AMP stimulates somatostatin gene transcription by phosphorylation of CREB at $\mathrm{Ser}^{133}$. Cell 59:675-680.

Green DJ, Gillette R (1982) Circadian rhythm of firing rate recorded from single cells in the rat suprachiasmatic slice. Brain Res 245:198-200.

Haby C, Lisovoski F, Aunis D, Zwiller J (1994) Stimulation of the cGMP pathway by $\mathrm{NO}$ induces expression of the immediate early genes c-fos and jun B in PC-12 cells. J Neurochem 62:496-501.

Hope BT, Michael GJ, Knigge KM (1991) NADPH-diaphorase is a nitric oxide synthase. Proc Natl Acad Sci USA 88:2811-2814.

Hunter T, Karin M (1992) The regulation of transcription by phosphorylation. Cell 70:375-387.

Inouye S, Kawamura H (1979) Persistence of circadian rhythmicity in a mammalian hypothalamic "island" containing the suprachiasmatic nucleus. Proc Natl Acad Sci USA 76:5962-5966.

Inouye S, Kawamura H (1982) Characteristics of a circadian pacemaker in the suprachiasmatic nucleus. J Comp Physiol 146:153-160.

Johnson RF, Morin LP, Moore RY (1988) Retinohypothalamic projections in the hamster and rat demonstrated using cholera toxin. Brain Res 462:301-312.

Kornhauser JM, Nelson DE, Mayo KE, Takahashi JS (1990) Photic and circadian regulation of c-fos gene expression in the hamster suprachiasmatic nucleus. Neuron 5:127-134.

Kornhauser JM, Nelson DE, Mayo KE, Takahashi JS (1992) Regulation of jun B messenger RNA and AP-1 activity by light and a circadian clock. Science 255:1581-1584.

Kovacs KJ, Sawchenko PE (1996) Sequence of stress-induced alterations in indices of synaptic and transcriptional activation in parvocellular neurosecretory neurons. J Neurosci 16:262-273.

Laemmli UK (1970) Cleavage of structural proteins during the assembly of the head of bacteriophage T4. Nature 227:680-685.

Mathur A, Golombek DA, Ralph MR (1996) cGMP-dependent kinase inhibitors block light-induced phase advances of circadian rhythms in vivo. Am J Physiol 270:R1031-R1036.
Meijer JH, Rietveld WJ (1989) Neurophysiology of the suprachiasmatic nucleus. Physiol Rev 69:671-707.

Mikkelsen JD, Larsen PJ, Ebling FJ (1993) Distribution of $N$-methyl-Daspartate (NMDA) receptor mRNAs in the rat suprachiasmatic nucleus. Brain Res 632:329-333.

Mize RR (1994) Quantitative image analysis for immunocytochemistry and in situ hybridization. J Neurosci Methods 54:219-237.

Montarolo PG, Goelet P, Castellucci VF, Morgan J, Kandel ER, Schacher S (1986) A critical period for macromolecular synthesis in long-term heterosynaptic facilitation in Aplysia. Science 234:1249-1254.

Moore RY, Lenn NL (1972) A retinohypothalamic projection in the rat. J Comp Neurol 146:1-14.

Morgan JI, Curran T (1989) Stimulus-transcription coupling in neurons: role of cellular immediate-early genes. Trends Neurosci 12:459-462.

Morin LP (1994) The circadian visual system. Brain Res Rev 67:102-127.

Morrissey JH (1981) Silver stain for protein in polyacrylamide gels: a modified procedure with enhanced uniform sensitivity. Anal Biochem 117:307-310.

Peunova N, Enlkolopov G (1993) Amplification of calcium-induced gene transcription by nitric oxide in neuronal cells. Nature 364:450-453.

Prosser RA, Gillette MU (1989) The mammalian circadian clock in the suprachiasmatic nuclei is reset in vitro by cAMP. J Neurosci 9:1073-1081.

Rea MA (1989) Light increases Fos-related protein immunoreactivity in the rat suprachiasmatic nuclei. Brain Res Bull 23:577-581.

Reuss S, Decker K, Rosseler L, Layes E, Schollmayer A, Spessert R (1995) Nitric oxide synthase in the hypothalamic suprachiasmatic nucleus of rat-evidence from histochemistry, immunochemistry and Western blot-and colocalization with VIP. Brain Res 695:257-262.

Rusak B, Robertson HA, Wisden W, Hunt SP (1990) Light pulses that shift rhythms induce gene expression in the suprachiasmatic nucleus. Science 248:1237-1240.

Sakamoto KM, Bardeleben C, Yates KE, Raines A, Golde DW, Gasson JC (1991) 5'-upstream sequence and the genomic structure of the human primary responsive gene EGR-1/TIS8. Oncogene 6:867-871.

Sassone-Corsi P, Visvader J, Ferland L, Mellon PL, Verma IM (1988) Induction of proto-oncogene fos transcription through adenylate cyclase pathway: characterization of cAMP-responsive element. Genes Dev 2:1529-1538.

Sauerwald A, Hoesche C, Oschwald R, Kilimann MW (1990) 5'-flanking region of the synapsin I gene: a $\mathrm{G}+\mathrm{C}$ rich, TATA- and CAAT-less, phylogenetically conserved sequence with cell type-specific promoter function. J Biol Chem 265:14932-14937.

Sheng M, Greenberg ME (1990) The regulation and function of c-fos and other immediate-early genes in the nervous system. Neuron 4:477-485.

Sheng M, Dougan ST, McFadden G, Greenberg ME (1988) Calcium and growth factor pathways of c-fos transcriptional activation require distinct upstream regulatory sequences. Mol Cell Biol 8:2787-2786.

Sheng M, McFadden G, Greenberg ME (1990) Membrane depolarization and calcium induce c-fos transcription via phosphorylation of transcription factor CREB. Neuron 4:571-582.

Sheng M, Thompson MA, Greenberg ME (1991) CREB: a $\mathrm{Ca}^{2+}$. regulated transcription factor phosphorylated by calmodulin-dependent kinase. Science 252:1427-1430.

Shibata S, Watanabe A, Hamada T, Ono M, Watanabe S (1994) $N$-methyl-D-aspartate induces phase shifts circadian rhythm of neuronal activity of rat SCN in vitro. Am J Physiol 267:R360-R364.

Shirakawa T, Moore RY (1994) Glutamate shifts the phase of the circadian neuronal firing rhythm in the rat suprachiasmatic nucleus in vitro. Neurosci Lett 178:47-50.

Shiromani PJ, Magner M, Winston S, Charness ME (1995) Time course of phosphorylated CREB and Fos-like immunoreactivity in the hypothalamic supraoptic nucleus. Mol Brain Res 29:163-171.

Summers TL, Ferraro JS, McCormack CE (1984) Phase-response and Aschoff illuminance curves for locomotor activity rhythm of the rat. Am J Physiol 246:R299-R304.

Sumova A, Ebling FJP, Maywood ES, Herbert J, Hastings MH (1994) Non-photic circadian entrainment in the Syrian hamster is not associated with phosphorylation of the transcriptional regulator CREB within the suprachiasmatic nucleus, but is associated with adrenocortical activation. Neuroendocrinology 59:579-589.

Takahashi JS, Zatz M (1982) Regulation of circadian rhythmicity. Science 217:1104-1111.

Takeuchi J, Shannon W, Aronin N, Schwartz WJ (1993) Compositional changes of AP-1 DNA-binding proteins are regulated by light in a mammalian circadian clock. Neuron 11:825-836. 
Tully T, Preat T, Boynton SC, Del Vecchio M (1994) Genetic dissection of consolidated memory in Drosophila. Cell 79:35-47.

Vincent SR, Kimura H (1992) Histochemical mapping of nitric oxide synthase in the rat brain. Neuroscience 46:755-784.

Watanabe A, Hamada T, Shibata S, Watanabe S (1994) Effects of nitric oxide synthase inhibitors on $N$-methyl-D-aspartate-induced phase delay of circadian rhythm of neuronal activity in the rat suprachiasmatic nucleus in vitro. Brain Res 646:161-164.

Weber ET, Gannon RL, Michel AM, Gillette MU, Rea MA (1995a) Nitric oxide synthase inhibitor blocks light-induced phase shifts of the circadian activity rhythm, but not c-fos expression in the suprachiasmatic nucleus of the Syrian hamster. Brain Res 692:137-142.
Weber ET, Gannon RL, Rea MA (1995b) cGMP-dependent protein kinase inhibitor blocks light-induced phase advances of circadian rhythms in vivo. Neurosci Lett 197:227-230.

Wollnik F, Brysch W, Uhlmann E, Gillardon F, Bravo R, Zimmerman M, Schingensiepen KH, Herdegen T (1995) Block of c-fos and jun B expression by antisense oligonucleotides inhibits light-induced phase shifts of the mammalian circadian clock. Eur J Neurosci 7:388-393.

Yin JCP, Wallach JS, Del Vecchio M, Wilder EL, Zhou H, Quinn WG, Tully $\mathrm{T}$ (1994) Induction of a dominant-negative CREB transgene blocks long-term memory in Drosophila. Cell 79:49-58.

Yin JCP, Del Vecchio M, Zhou H, Tully T (1995) CREB as a memory modulator: induced expression of a dCREB2 activator isoform enhances long-term memory in Drosophila. Cell 81:107-115. 